\section{RICYDE. Revista Internacional de Ciencias del Deporte doi: $10.5232 /$ ricyde \\ Rev. Int. cienc. deporte}

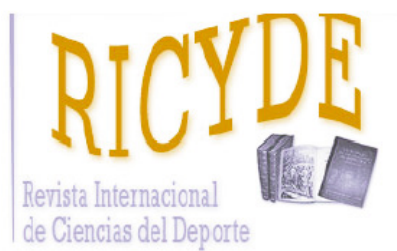

RICYDE. Revista Internacional de Ciencias del Deporte Volume XV - Year XV

Pages: 235-248 - ISSN: $1885-3137$ Issue 57 - July 2019

\title{
Evaluación de la condición física del jugador de tenis en silla de ruedas de alto nivel según nivel competitivo y tipo de lesión \\ Assessment of the physical condition of the high-level wheelchair tennis player according to competitive level and kind of injury
}

\author{
Alejandro Sánchez-Pay ${ }^{1}$, David Sanz-Rivas²
}

1. Facultad de Ciencias del Deporte. Universidad de Murcia. España

2. Real Federación Española de Tenis. España

\section{Resumen}

Evaluar la condición física de los deportistas permite conocer qué aspectos deben ser mejorados, así como comparar el nivel de condición física del jugador respecto a grupos de referencia. El objetivo de este estudio fue medir los niveles de condición física de jugadores de tenis en silla de ruedas (TSR) y establecer comparaciones en función del ranking y tipo de lesión. Los participantes de esta investigación fueron 9 jugadores masculinos de TSR de alto nivel. Los jugadores completaron una batería de tests que constó de las siguientes pruebas: fuerza del antebrazo, velocidad de servicio, velocidad de desplazamiento en 5,10 , y $20 \mathrm{~m}$ (con y sin raqueta), test de agilidad (con y sin raqueta), test de lanzamiento de balón medicinal (golpes de saque, derecha, y revés) y un test incremental de resistencia específico de TSR. Los resultados mostraron que los jugadores de nivel más alto tienen mejores niveles de condición física en todas las pruebas, encontrando diferencias significativas en casi todos los tests. Los jugadores con una limitación funcional mayor mostraron valores más bajos, aunque no se encontraron gran cantidad de diferencias estadísticamente significativas. Los test utilizados pueden ser empleados para evaluar los niveles de condición física en jugadores de TSR pues discriminan en función del nivel del deportista, y no son excluyentes en relación a la limitación funcional.

Palabras clave: deporte paralímpico; valoración física; test de campo; tenis en silla de ruedas.

\begin{abstract}
Testing the physical condition of athletes allows to know which aspects should be improved on the player, as well as the level of physical condition of the player with respect to normative groups, among others. The aim of this study was to test the fitness levels of wheelchair tennis (WT) players and establish comparisons based on their ranking and their level of impairment. The participants in this research were 9 male high-level WT players. The players completed a battery of tests consisting in the following tests: forearm strength, speed of service, sprint in 5, 10, and $20 \mathrm{~m}$ (with and without racket), agility test (with and without racket), medicine ball throw test (serve, forehand, and backhand movements) and an endurance specific wheelchair tennis test. The results showed that the highest-level players have better fitness levels in all the tests, finding significant differences in almost all of them. The players with greater functional limitation showed lower values, although a large number of statistically significant differences were not found. This test battery used can be used to assess the fitness levels of WT players because they discriminate depending on the level of the athlete, and are not exclusive in relation to their activity limitation.
\end{abstract}

Key words: paralympic sport; physical fitness; field test; wheelchair tennis.

Correspondencia/correspondence: Alejandro Sánchez-Pay

Facultad de Ciencias del Deporte. Universidad de Murcia. España

Email: aspay@um.es 
Sánchez-Pay, A.; Sanz-Rivas, D. (2019). Evaluación de la condición física del jugador de tenis en silla de ruedas de alto nivel según nivel competitivo y tipo de lesión. RICYDE. Revista internacional de ciencias del deporte. 57(15), 235-248. https://doi.org/10.5232/ricyde2019.05702

\section{Introducción}

$\mathrm{D}$ urante las últimas décadas ha existido un rápido crecimiento del deporte paralímpico, con un incremento del número de países y atletas que han participado en los Juegos Paralímpicos (Bernardi y col., 2010). El deporte para personas con discapacidad presenta actualmente, entre otras, una clara orientación hacia el deporte competitivo y de alto rendimiento, y ha ganado popularidad, especialmente en juegos de pelota como baloncesto en silla de ruedas, rugby en silla de ruedas o el tenis en silla de ruedas. El tenis en silla de ruedas (TSR) se ha establecido como uno de los deportes paralímpicos favoritos para el público (Diaper y Goosey-Tolfrey, 2009) y entre los cuatro más importantes dentro del programa de los Juegos Paralímpicos de verano (Abel, Platen, Rojas Vega, Schneider, y Strüder, 2008).

El jugador de TSR debe realizar movimientos específicos con su silla de ruedas que incluyen el arranque, sprint, frenado y giro (Sanz, 2003), y todo ello mientras se sostiene una raqueta con la mano (Goosey-Tolfrey y Moss, 2005). Esta secuencia hace que la movilidad sea considerada como un importante factor de éxito en el TSR (Bullock y Pluim, 2003). El rendimiento en la movilidad depende de la combinación de factores asociados al deportista, la silla, y la relación entre ambos (Mason, Van Der Woude, y Goosey-Tolfrey, 2013). Así, moverse correctamente permite al jugador prepararse adecuadamente para realizar un golpe con garantías de éxito (Filipčič y Filipčič, 2009a). Parece pues que la capacidad de realizar sprints de manera repetida, así como cambios de dirección, son muy importantes en el TSR, aspecto que ha sido de interés en el baloncesto en silla de ruedas (Granados y col., 2015), no encontrándose información al respecto en el TSR. Conocer estos datos aportaría a los entrenadores información útil para diseñar ejercicios de agilidad y movilidad específica, con alta implicación de la parte superior del cuerpo, así como recomendaciones de preparación física en TSR (Schuster, Howells, Robineau, y col., 2018).

Si bien la movilidad con la silla de ruedas parece ser uno de los factores más importantes para el rendimiento en el jugador de TSR, la situación de servicio parece no favorecer de igual forma que sucede en el tenis convencional (Sánchez-Pay, Torres-Luque, Cabello-Manrique, Sanz-Rivas, y Palao, 2015; Sánchez-Pay, Torres-Luque, Fernández-García, Sanz-Rivas, y Palao, 2017), hecho planteado por los estudios de Sánchez-Pay y col. $(2015,2017)$ a partir de las estadísticas de competición. Aunque la velocidad del servicio podría ser un indicador del nivel competitivo, sólo se tiene constancia de que la limitación funcional de los jugadores afecta a la velocidad de golpeo en el servicio (Cavedon, Zancanaro, y Milanese, 2014).

En el TSR existe gran cantidad de información relacionada con las demandas de la competición a nivel técnico (Sanchez-Pay y col., 2015, 2017), táctico (Filipčič y Filipčič, 2009b), físico (Ponzano y Gollin, 2017) y fisiológico (Croft, Dybrus, Lenton, y GooseyTolfrey, 2010; Roy, Menear, Schmid, Hunter, y Malone, 2006; Sánchez-Pay, Torres-Luque, y Sanz-Rivas, 2015). A pesar de que la mayoría de las investigaciones han utilizado pruebas de laboratorio para evaluar la condición física y el rendimiento (Goosey-Tolfrey y Leicht, 2013), los test de campo se convierten, de igual forma, en una opción fiable para establecer el nivel de rendimiento de los jugadores (De Groot, Balvers, Kouwenhoven, y Janssen, 2012). Sin embargo, son escasas las investigaciones que muestren valores sobre la condición física de los jugadores de TSR. En otros deportes paralímpicos como el baloncesto en silla de ruedas existen estudios que valoran la condición física de los jugadores por medio de test de campo, mostrando diferencias a nivel competitivo (De Groot y col., 2012) a nivel de limitación funcional (Almena, Pérez-Tejero, Coterón, y Veiga, 2015; Gil, Yanci, Otero, y col., 2015, Yanci, Iturricastillo, Lozano, y Granados, 2015). Por lo tanto, el objetivo de este estudio será conocer el nivel de condición física del jugador de tenis en silla de ruedas, así como 
Sánchez-Pay, A.; Sanz-Rivas, D. (2019). Evaluación de la condición física del jugador de tenis en silla de ruedas de alto nivel según nivel competitivo y tipo de lesión. RICYDE. Revista internacional de ciencias del deporte. 57(15), 235-248. https://doi.org/10.5232/ricyde2019.05702

establecer posibles diferencias en función del nivel competitivo y la limitación funcional de los jugadores.

\section{Método}

\section{Participantes}

Los participantes de la investigación fueron los 9 mejores jugadores de tenis en silla de ruedas masculinos a nivel nacional al comienzo del año 2018, con edades comprendidas entre los 18 y 50 años (38.35 \pm 11.28 años). De los 9 jugadores participantes en el estudio, los 4 mejores (dos jugadores top 20 ITF ranking y 2 top 50 ITF ranking) formaban el grupo de la Selección Nacional (Grupo 1), todos ellos deportistas de alto nivel y seleccionables para los Juegos Paralímpicos por su clasificación. Los 5 jugadores restantes mejor clasificados formaron el segundo grupo (Grupo 2). Además, los participantes fueron agrupados en dos grupos en función del tipo de lesión: a) deportistas con una lesión medular (LM), y b) deportistas con otro tipo de lesión (no lesión medular; no LM) tal y como se ha realizado en estudios previos (Gil y col., 2015). En la tabla 1 se puede observar las características de la muestra.

Tabla 1. Características de los jugadores tenis en silla de ruedas.

\begin{tabular}{|c|c|c|c|c|c|c|c|c|c|}
\hline Jugador & $\begin{array}{c}\text { Ranking } \\
\text { nacional }\end{array}$ & $\begin{array}{c}\text { Ranking } \\
\text { internacional }\end{array}$ & $\begin{array}{c}\text { Grupo } \\
\text { nivel }\end{array}$ & Lesión & $\begin{array}{c}\text { Tipo } \\
\text { de } \\
\text { lesión }\end{array}$ & $\begin{array}{c}\text { Edad } \\
\text { (años) }\end{array}$ & $\begin{array}{c}\text { Peso } \\
\text { (kg) }\end{array}$ & $\begin{array}{c}\text { Entreno } \\
\text { semanal } \\
\text { (horas) }\end{array}$ & $\begin{array}{c}\text { Experiencia } \\
\text { tenis (años) }\end{array}$ \\
\hline 1 & 1 & Top 20 & 1 & At & $\begin{array}{c}\text { No } \\
\text { LM }\end{array}$ & 24 & 61 & 20 & 12 \\
\hline 2 & 2 & Top 20 & 1 & At & $\begin{array}{c}\text { No } \\
\text { LM }\end{array}$ & 18 & 65 & 15 & 8 \\
\hline 3 & 3 & Top 50 & 1 & L2 & LM & 34 & 67 & 8 & 5 \\
\hline 4 & 7 & Top 50 & 1 & OI & $\begin{array}{c}\text { No } \\
\text { LM }\end{array}$ & 45 & 57 & 3 & 24 \\
\hline 5 & 5 & Top 100 & 2 & Af & $\begin{array}{c}\text { No } \\
\text { LM }\end{array}$ & 39 & 52 & 6 & 9 \\
\hline 6 & 6 & Top 100 & 2 & D11 & LM & 35 & 72 & 10 & 2 \\
\hline 7 & 8 & Top 100 & 2 & Af & $\begin{array}{c}\text { No } \\
\text { LM }\end{array}$ & 50 & 73 & 8 & 17 \\
\hline 8 & 9 & Top 150 & 2 & D9 & LM & 50 & 70 & 6 & 8 \\
\hline 9 & 10 & Top 150 & 2 & D4 & LM & 41 & 57 & 8 & 7 \\
\hline
\end{tabular}

Nota: At: Amputación a nivel tibial. Af: Amputación a nivel femoral. L: Afectación medular a nivel lumbar. D: Afectación medular a nivel dorsal. OI: Osteogénesis imperfecta. LM: Lesión medular. No LM: No lesión medular.

\section{Procedimiento}

Todos los deportistas fueron informados sobre el procedimiento del estudio, y entregaron un consentimiento informado para participar en el mismo. Además, el comité ético de la Federación Española de Tenis aprobó dicho estudio. Se citó a todos los jugadores que iban a participar en las pruebas a la misma hora del día. Tras un calentamiento estandarizado de 10 min, los jugadores completaron los test de condición física en el siguiente orden. Día 1: Test de velocidad $(5,10$ y $20 \mathrm{~m})$, test de agilidad (T-test), test de velocidad del servicio, y test de lanzamiento de balón medicinal (derecha, revés y servicio). Los jugadores se dividieron en 
Sánchez-Pay, A.; Sanz-Rivas, D. (2019). Evaluación de la condición física del jugador de tenis en silla de ruedas de alto nivel según nivel competitivo y tipo de lesión. RICYDE. Revista internacional de ciencias del deporte. 57(15), 235-248. https://doi.org/10.5232/ricyde2019.05702

grupos que iban pasando por las diferentes estaciones para realizar las pruebas. Día 2: test de resistencia incremental (Hit and Turn Tennis Test) y dinamometría manual. Todas las pruebas se realizaron a partir de las 10:00 horas hasta las 11:30 horas. Los datos se recogieron a través de una hoja de registro durante el desarrollo de las pruebas. Las pruebas tuvieron lugar en pistas cubiertas de superficie dura, coincidiendo con el final del periodo de pretemporada de todos los jugadores (enero, 2018).

\section{Instrumentos}

Se registraron tres medidas por participante a través de test de campo ampliamente utilizados en la evaluación de tenistas y jugadores en silla de ruedas debido a su fiabilidad y validez ecológica (De Groot y col., 2012; Gil y col., 2015, Granados y col., 2015; Sánchez-Pay, Torres-Luque, y Palao, 2011):

$\Rightarrow$ Test de velocidad de desplazamiento: La velocidad de desplazamiento se midió a través de Chronojump Photecell ${ }^{\circledR}$ (Chronojump, Barcelona, España) y el software Cronojump versión 1.7.1.8 para MAC. Se utilizaron 4 puertas situadas a $0,5,10$, y $20 \mathrm{~m}$ (figura 1) con una altura de $0,5 \mathrm{~m}$ respecto al suelo. Los sujetos partieron desde una línea a $0,5 \mathrm{~m}$ detrás de la primera puerta. Cada participante realizó el test tres veces sin raqueta, y tres veces con raqueta, con un tiempo de descanso entre cada repetición de $2 \mathrm{~min}$. Se registró el mejor valor de la carrera de $20 \mathrm{~m}$ de 3 intentos. El tiempo se contabilizó en segundos (s) y milésimas de segundo (ms) con un error de $\pm 0.001 \mathrm{~s}$. El coeficiente de variación (CV) para este test entre las distintas repeticiones realizadas fue de $2,56 \%$.

$\Rightarrow$ Test de agilidad (T-Test). El participante se situó en el centro de la pista detrás de la línea de fondo, y debían desplazarse a las intersecciones de la línea de individuales con la línea de servicio, pasando siempre por la zona central de la pista (T) hasta volver a la zona de partida (figura 2). Cada participante realizó el test tres veces sin raqueta, y tres veces con raqueta, con un tiempo de descanso entre cada repetición de 2 min. Se registró el mejor valor de tres intentos. El tiempo se midió a través de Chronojump Photecell ${ }^{\circledR}$ (Chronojump, Barcelona, España) y el software Cronojump versión 1.7.1.8 para MAC con una puerta situada en la línea de fondo para registrar el inicio y el final de la prueba. El CV para este test entre las distintas repeticiones realizadas fue de a 1,85\%.

$\Rightarrow$ Velocidad de servicio: Para medir la velocidad del servicio se utilizó una pistola de radar (Stalker Pro Inc., Plano, Texas, USA) con una frecuencia de registro de datos de $33 \mathrm{hz}$. Al jugador se le pidió que realizase 10 servicios a la máxima velocidad dirigidos a la zona abierta del cuadro de saque (intersección entre la línea de individuales y la línea de servicio) desde el lado de la ventaja para los jugadores diestros, y desde el lado de los iguales al jugador zurdo. El radar fue dispuesto detrás del jugador a la misma altura de golpeo y orientado en la misma dirección de la pelota. El radar mide con un rango de velocidades comprendidas entre 1 y $480 \mathrm{~km} / \mathrm{h}$ con una precisión de $\pm 0,16 \mathrm{~km} / \mathrm{h}$. Se registró el valor promedio de 10 saques en $\mathrm{km} / \mathrm{h}$.

$\Rightarrow$ Fuerza del tren superior: Se evaluó la fuerza explosiva a través de tres pruebas de lanzamiento de balón medicinal de $2 \mathrm{~kg}$, simulando los golpeos de derecha, revés y servicio. Los participantes se situaron detrás de la línea de lanzamiento con una posición de $45^{\circ}$ respecto a la misma. Perpendicular a la línea de lanzamiento se dispuso una cinta métrica de $15 \mathrm{~m}$ de longitud sobre el suelo y dos evaluadores marcaban la zona de bote de la pelota registrado en tramos de $0,10 \mathrm{~m}$. Los participantes realizaron cada tipo de lanzamiento tres veces, con un tiempo de descanso entre cada repetición de $2 \mathrm{~min}$. Se registró el mejor valor de tres intentos. La técnica de lanzamiento simulando cada gesto consistía en: 
Sánchez-Pay, A.; Sanz-Rivas, D. (2019). Evaluación de la condición física del jugador de tenis en silla de ruedas de alto nivel según nivel competitivo y tipo de lesión. RICYDE. Revista internacional de ciencias del deporte. 57(15), 235-248. https://doi.org/10.5232/ricyde2019.05702

- Lanzamiento derecha/revés: El balón debía mantenerse con ambas manos por el lado de lanzamiento (derecha o revés). La mano del lado de lanzamiento realizaba la fuerza, mientras que la mano contraria al lado de lanzamiento hacía de guía. A la señal del evaluador, el jugador debía realizar un movimiento explosivo y lanzar el balón con ambas manos lo más lejos posible. El CV para el test de derecha entre las distintas repeticiones realizadas fue de $3,17 \%$ y en el test de revés de $4,02 \%$.

- Lanzamiento de servicio: El balón debía mantenerse apoyado sobre la palma de la mano dominante a un lado de la cabeza. A la señal del evaluador, el jugador debía realizar un movimiento explosivo y lanzar el balón simulando un lanzamiento de peso en atletismo. El CV en este test entre las distintas repeticiones realizadas fue de $0,92 \%$.

$\Rightarrow$ Dinamometría manual: Se realizó la prueba de dinamometría manual con el objeto de valorar la fuerza máxima isométrica en los flexores de los dedos. Para ello se utilizó un dinamómetro Smedley III T-18A (Takei, Tokyo, Japón) con un rango comprendido entre 0 y $100 \mathrm{~kg}$ con incrementos de $1 / 2 \mathrm{~kg}$ y una precisión de $\pm 2 \mathrm{~kg}$. La prueba se realizó con el brazo extendido y pegado a la rueda sin llegar a contactar con ella. Cada sujeto realizó tres intentos máximos con cada mano tras una fase de familiarización con el instrumento con repeticiones sub-máximas. El tiempo de descanso entre cada intento fue de $2 \mathrm{~min}$. Se registró el mejor valor de tres intentos en $\mathrm{N} \cdot \mathrm{kg}^{-1}$. El CV para este test entre las distintas repeticiones realizadas fue de a $1,37 \%$.

$\Rightarrow$ Test de resistencia incremental (Hit and Turn Tennis Test): El objetivo del test es evaluar la resistencia anaeróbica específica del jugador a través del nivel alcanzado. Este test es una adaptación del desarrollado para el tenis convencional por Ferrauti, Kinner, y Fernández-Fernández (2011). El test consiste en simular un golpeo encima de un cono situado en la intersección de la línea de dobles con la de fondo coincidiendo con las señales sonoras que emite un CD. Tras ese golpeo, el jugador debe simular otro golpeo en el lado opuesto y así hasta terminar la serie. En esta adaptación, el golpeo debía realizarse próximo a un cono situado entre la intersección de la línea de individual con la de dobles, disminuyendo así la distancia de desplazamiento para el jugador en TSR. Cada serie aumenta el número de golpeos (de 12 a 16) y la duración de la misma (de 47 a $50 \mathrm{~s}$ ), disminuyendo el tiempo entre cada golpeo (de 4,9 s en el primer nivel a $3 \mathrm{~s}$ en el nivel 20). El test finaliza cuando el jugador no es capaz de llegar al cono al ritmo marcado por las señales sonoras (figura 3). Para escuchar las señales sonoras emitidas se utilizó un altavoz Megaboom (Logitech, Lausanne, Suiza). Se registró el periodo alcanzado por cada jugador cuando éste ya no era capaz de simular el golpeo en la zona señalada al mismo tiempo que sonaba la señal acústica.

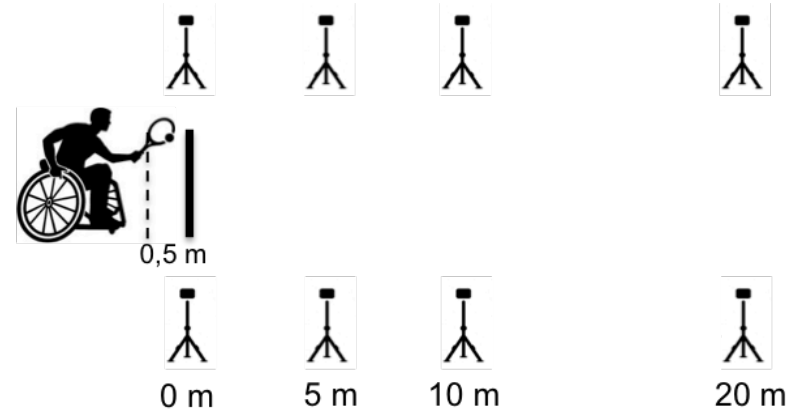

Figura 1. Test de velocidad 5, 10, y 20 metros. 


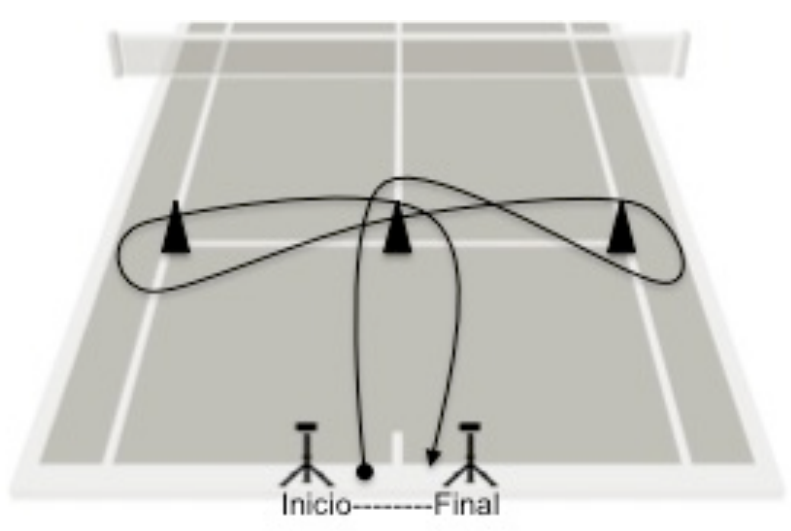

Figura 2. Test de agilidad T-Test.

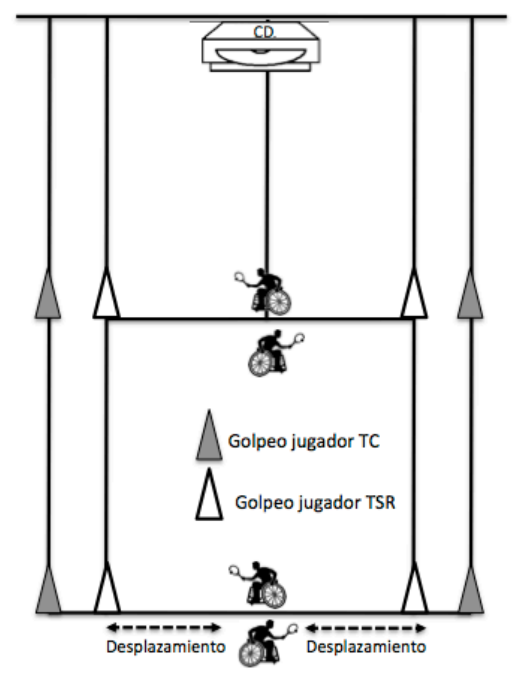

Figura 3. Adaptación del test de resistencia incremental (Hit and Turn Tennis Test) propuesto por Ferrauti y col. (2011).

\section{Análisis estadístico}

Se realizó un análisis descriptivo de los datos que incluyó el cálculo de media y desviación típica $(\mathrm{M} \pm \mathrm{DT})$ de las variables estudiadas. Debido al pequeño tamaño de la muestra se utilizaron los tests de Shapiro-Wilk y Levene para contrastar la normalidad y homogeneidad de varianzas para cada variable. Todas la variables obtuvieron valores de $p>0.05$ excepto en la dinamometría con el brazo dominante. Se aplicó la prueba T de Student y U de MannWhitney para la comparación de medias entre grupos en función de la normalidad de cada variable, considerando un nivel de significación de $\mathrm{p}<.05$. Se calculó el tamaño del efecto (d) a través de la g de Hedges debido al tamaño muestral (Hedges y Olkin, 1985). Para determinar la magnitud del efecto se siguieron las recomendaciones de Rhea (2004) para deportistas entrenados, siendo $<0.25$ considerado como trivial; $0.25-0.50$ bajo; 0-50-1.0 
Sánchez-Pay, A.; Sanz-Rivas, D. (2019). Evaluación de la condición física del jugador de tenis en silla de ruedas de alto nivel según nivel competitivo y tipo de lesión. RICYDE. Revista internacional de ciencias del deporte. 57(15), 235-248. https://doi.org/10.5232/ricyde2019.05702

moderado; $y>1$ alto. Además, se calculó la diferencia porcentual de cada test entre los dos grupos de nivel (grupo 1 y grupo 2). Los análisis fueron realizados con el software SPSS para Windows (Versión 20.0. Armonk, NY: IBM Corp.)

\section{Resultados}

La tabla 2 muestra la comparación de las puntuaciones en los test en función del nivel de los deportistas. Los jugadores con mejor ranking (grupo 1) mostraron mejores puntuaciones en todos los tests excepto en la dinamometría no dominante. Se encontraron diferencias estadísticamente significativas en los test de $5 \mathrm{~m}$ sin raqueta $\left(\mathrm{p}=.047, \mathrm{~d}_{\mathrm{g}}=1,43\right), 10$ metros $\sin$ raqueta $\left(\mathrm{p}=.009, \mathrm{~d}_{\mathrm{g}}=2,15\right), 20 \mathrm{~m}$ sin raqueta $\left(\mathrm{p}=.009, \mathrm{~d}_{\mathrm{g}}=2,11\right), 5 \mathrm{~m}$ con raqueta $(\mathrm{p}=$ $\left..041, \mathrm{~d}_{\mathrm{g}}=1,51\right), 20 \mathrm{~m}$ con raqueta $\left(\mathrm{p}=.027, \mathrm{~d}_{\mathrm{g}}=1,64\right)$, T-test con raqueta $\left(\mathrm{p}=.034, \mathrm{~d}_{\mathrm{g}}=\right.$ $1,57)$, lanzamiento de balón de servicio $\left(\mathrm{p}=.029, \mathrm{~d}_{\mathrm{g}}=1,64\right)$ y en la prueba de resistencia Hit and Turn $\left(\mathrm{p}=.002, \mathrm{~d}_{\mathrm{g}}=2,94\right)$.

Tabla 2. Diferencias de la condición física en función de los grupos de nivel.

\begin{tabular}{|l|c|c|c|c|c|c|}
\hline & $\begin{array}{c}\text { Total } \\
(\mathrm{n}=9)\end{array}$ & $\begin{array}{c}\text { Grupo } 1 \\
(\mathrm{n}=4)\end{array}$ & $\begin{array}{c}\text { Grupo } 2 \\
(\mathrm{n}=5)\end{array}$ & & & \\
\hline & $\mathrm{M}(\mathrm{DT})$ & $\mathrm{M}(\mathrm{DT})$ & $\mathrm{M}(\mathrm{DT})$ & $\mathrm{p}$ & Dif. & $\mathrm{d}_{\mathrm{g}}$ \\
\hline Dina. Dom. (kg) & $46,33(4,18)$ & $46,38(4,11)$ & $46,3(4,72)$ & 0,981 & 0,08 & 0,02 \\
\hline Dina. No Dom. (kg) & $38,61(6,27)$ & $37,25(7,15)$ & $39,7(6,08)$ & 0,595 & $-2,45$ & 0,33 \\
\hline V. saque (km/h) & $114,9(10,07)$ & $120,65(11,15)$ & $110,3(7,07)$ & 0,132 & 10,35 & 1,02 \\
\hline 5m sin raqueta (s) & $1,57(0,14)$ & $1,44(0,1)$ & $1,65(0,15)$ & 0,047 & $-0,21$ & 1,43 \\
\hline 10m sin raqueta (s) & $2,88(0,3)$ & $2,74(0,13)$ & $3,16(0,2)$ & 0,009 & $-0,42$ & 2,15 \\
\hline 20m sin raqueta (s) & $5,62(0,64)$ & $4,98(0,32)$ & $5,74(0,32)$ & 0,009 & $-0,76$ & 2,11 \\
\hline 5m con raqueta (s) & $1,71(0,2)$ & $1,5(0,1)$ & $1,78(0,2)$ & 0,041 & $-0,28$ & 1,51 \\
\hline 10m con raqueta (s) & $3,2(0,35)$ & $3(0,48)$ & $3,04(0,33)$ & 0,885 & $-0,04$ & 0,09 \\
\hline 20m con raqueta (s) & $5,88(0,64)$ & $5,06(0,39)$ & $5,77(0,38)$ & 0,027 & $-0,71$ & 1,64 \\
\hline T-Test sin raqueta (s) & $12,59(1,01)$ & $11,76(0,83)$ & $12,96(0,81)$ & 0,065 & $-1,20$ & 1,30 \\
\hline T-Test con raqueta (s) & $12,85(1,24)$ & $11,79(0,77)$ & $13,39(1)$ & 0,034 & $-1,60$ & 1,57 \\
\hline Lanz. Balón D (m) & $5,71(1,87)$ & $6,8(1,59)$ & $5,2(2,05)$ & 0,242 & 1,60 & 0,76 \\
\hline Lanz. Balón R (m) & $5,51(1,81)$ & $6,64(1,92)$ & $5,16(1,6)$ & 0,248 & 1,48 & 0,75 \\
\hline Lanz. Balón S (m) & $6,88(1,39)$ & $8,25(1,26)$ & $6,3(0,88)$ & 0,029 & 1,95 & 1,64 \\
\hline Hit and Turn & $15,22(2,99)$ & $18(1,41)$ & $13(1,58)$ & 0,002 & 5,00 & 2,94 \\
\hline
\end{tabular}

Valores medios (M) y desviación típica (DT). Dina: Dinamometría, Dom: Dominante, V: Velocidad, Lanz: Lanzamiento, D: Derecha, R: Revés, S: Saque, Dif. Diferencia de medias, d: tamaño del efecto.

La tabla 3 muestra la comparación de las puntuaciones en los test en función del tipo de lesión del deportista (no LM y LM). Los jugadores con una lesión medular mostraron peores valores en todas las pruebas físicas. Se encontraron diferencias estadísticamente significativas en la dinamometría dominante $\left(\mathrm{p}=.036, \mathrm{~d}_{\mathrm{g}}=1,54\right), 5 \mathrm{~m}$ sin raqueta $\left(\mathrm{p}=.010, \mathrm{~d}_{\mathrm{g}}=2,18\right), 20$ $\mathrm{m}$ con raqueta $\left(\mathrm{p}=.037, \mathrm{~d}_{\mathrm{g}}=1,54\right)$, T-Test con raqueta $\left(\mathrm{p}=.041, \mathrm{~d}_{\mathrm{g}}=1,49\right)$ y en la prueba de resistencia Hit and Turn $\left(\mathrm{p}=.034, \mathrm{~d}_{\mathrm{g}}=1,57\right)$. 
Sánchez-Pay, A.; Sanz-Rivas, D. (2019). Evaluación de la condición física del jugador de tenis en silla de ruedas de alto nivel según nivel competitivo y tipo de lesión. RICYDE. Revista internacional de ciencias del deporte. 57(15), 235-248. https://doi.org/10.5232/ricyde2019.05702

Tabla 3. Diferencias de la condición física en función del tipo de lesión.

\begin{tabular}{|c|c|c|c|c|c|}
\hline & $\begin{array}{l}\text { No LM } \\
(n=5)\end{array}$ & $\begin{array}{c}\text { LM } \\
(n=5)\end{array}$ & & & \\
\hline & $\mathrm{M}(\mathrm{DT})$ & $\mathrm{M}(\mathrm{DT})$ & $\mathrm{p}$ & Dif. & $\mathrm{dg}_{\mathrm{g}}$ \\
\hline Dina. Dom. (kg) & $48,8(4,16)$ & $43,25(0,87)$ & 0,036 & 5,55 & 1,54 \\
\hline Dina. No Dom. (kg) & $39,7(7,6)$ & $37,25(4,84)$ & 0,595 & 2,45 & 0,33 \\
\hline V. de saque $(\mathrm{km} / \mathrm{h})$ & $119,6(10,2)$ & $109,03(6,97)$ & 0,122 & 10,58 & 1,05 \\
\hline $5 \mathrm{~m}$ sin raqueta $(\mathrm{s})$ & $1,44(0,07)$ & $1,7(0,14)$ & 0,010 & $-0,26$ & 2,18 \\
\hline $10 \mathrm{~m} \sin$ raqueta $(\mathrm{s})$ & $2,86(0,25)$ & $3,13(0,25)$ & 0,150 & $-0,27$ & 0,96 \\
\hline $20 \mathrm{~m}$ sin raqueta (s) & $5,17(0,52)$ & $5,69(0,33)$ & 0,131 & $-0,52$ & 1,03 \\
\hline $5 \mathrm{~m}$ con raqueta $(\mathrm{s})$ & $1,55(0,15)$ & $1,79(0,22)$ & 0,104 & $-0,23$ & 1,16 \\
\hline $10 \mathrm{~m}$ con raqueta $(\mathrm{s})$ & $2,83(0,19)$ & $3,25(0,44)$ & 0,096 & $-0,42$ & 1,16 \\
\hline $20 \mathrm{~m}$ con raqueta $(\mathrm{s})$ & $5,15(0,38)$ & $5,84(0,42)$ & 0,037 & $-0,68$ & 1,54 \\
\hline T-Test sin raqueta (s) & $11,88(0,77)$ & $13,11(0,85)$ & 0,057 & $-1,23$ & 1,36 \\
\hline T-Test con raqueta (s) & $11,99(0,87)$ & $13,55(1,01)$ & 0,041 & $-1,56$ & 1,49 \\
\hline Lanz. Balón D (m) & $6,8(1,52)$ & $4,8(2)$ & 0,130 & 2,00 & 1,02 \\
\hline Lanz. Balón R (m) & $6,47(1,69)$ & $5(1,83)$ & 0,251 & 1,47 & 0,75 \\
\hline Lanz. Balón S (m) & $7,76(1,39)$ & $6,43(1,24)$ & 0,178 & 1,34 & 0,89 \\
\hline Hit and Turn & $17(2,35)$ & $13(2,16)$ & 0,034 & 4,00 & 1,57 \\
\hline
\end{tabular}

Valores medios (M) y desviación típica (DT). Dina: Dinamometría, Dom: Dominante, V: Velocidad, Lanz: Lanzamiento, D: Derecha, R: Revés, S: Saque, Dif: Diferencia de medias, d: tamaño del efecto, LM: Lesión medular. No LM: No lesión medular.

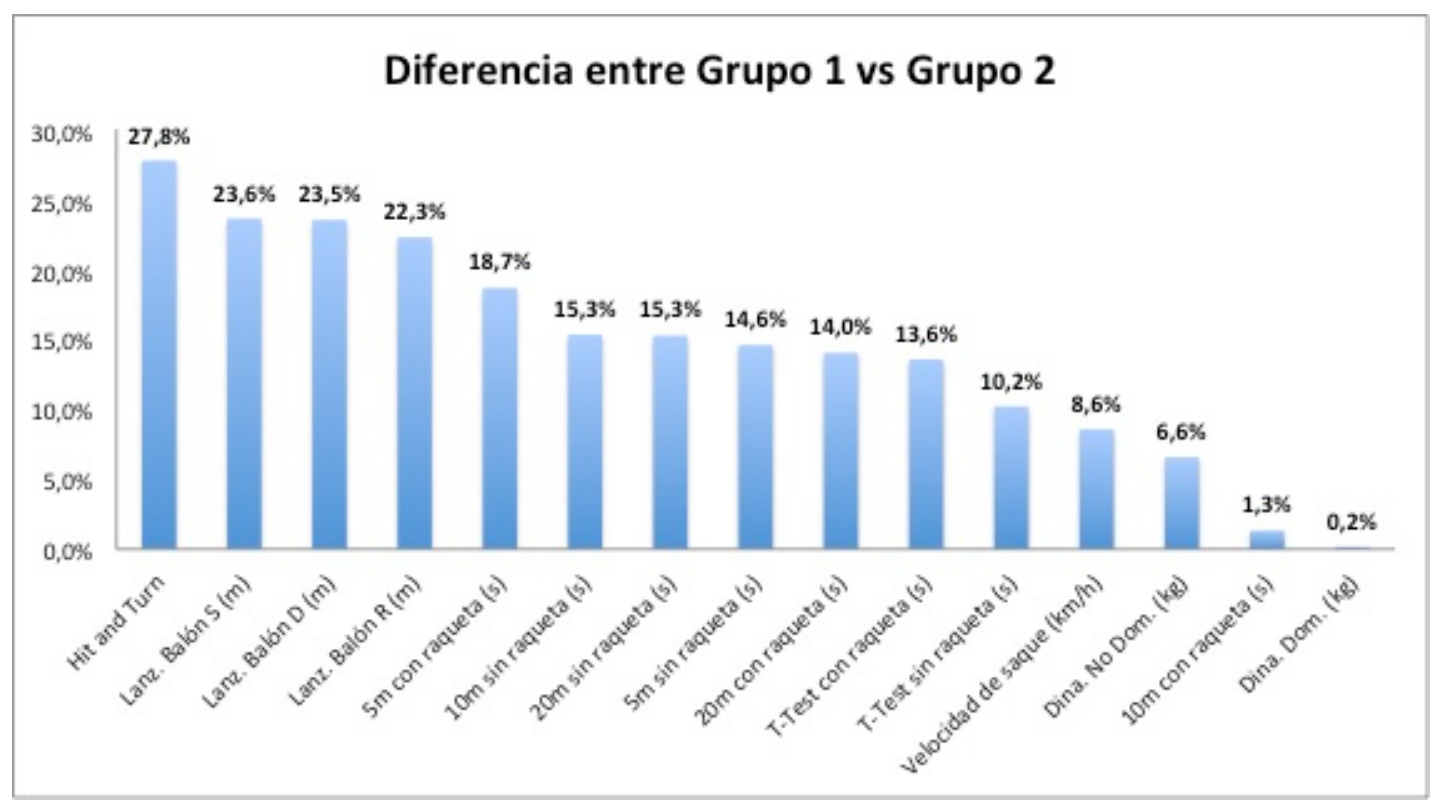

Figura 4. Diferencia porcentual de cada prueba entre los dos grupos de nivel. 
Sánchez-Pay, A.; Sanz-Rivas, D. (2019). Evaluación de la condición física del jugador de tenis en silla de ruedas de alto nivel según nivel competitivo y tipo de lesión. RICYDE. Revista internacional de ciencias del deporte. 57(15), 235-248. https://doi.org/10.5232/ricyde2019.05702

Las diferencias porcentuales entre los dos grupos de nivel sobre cada prueba se pueden observar en la figura 4. La prueba de resistencia es la que mayor diferencia representa entre ambos grupos, siendo un $27 \%$ menor los valores en los jugadores de nivel menor respecto a los de mayor nivel. Seguidamente, las diferencias porcentuales más altas se dan en las pruebas relacionadas con los lanzamientos (por encima del 20\%). A su vez, todas las pruebas de velocidad y agilidad (con y sin raqueta) muestran diferencias mayores, entre un $10 \%$ y un $20 \%$.

\section{Discusión}

Conocer los niveles de condición física de los jugadores ayuda a diseñar y planificar los entrenamientos de estos, así como observar las fortalezas y debilidades de los jugadores respecto a grupos de referencia (Roeter y Ellenbecker, 2008). El objetivo de esta investigación fue valorar los niveles de condición física de los mejores jugadores nacionales de tenis en silla de ruedas, y observar las posibles diferencias en función del ranking y el tipo de lesión. En líneas generales, se observan muchas pruebas con valores superiores para los jugadores mejor clasificados en el ranking. Además, los jugadores con lesión medular obtuvieron peores valores en todas las pruebas, aunque con menor número de diferencias significativas.

Los jugadores de TSR deben desplazar la silla, acelerando y frenando mientras mantienen una raqueta en la mano para después golpear la pelota. Tener unos niveles altos de fuerza en los músculos del antebrazo les permite desarrollar estas acciones de una forma más eficiente. Los valores medios mostrados por los jugadores de TSR en la dinamometría de la mano dominante son similares a los valores reportados por jugadores de baloncesto en silla de ruedas tanto en los estudios de Granados y col. (2015) y Gil y col. (2015), que se sitúan cercanos a $45 \mathrm{~kg}$, e inferiores a los descritos por Yanci y col. (2015) cercanos a $58 \mathrm{~kg}$. Se han encontrado diferencias estadísticamente significativas sobre la mano dominante en función del grupo de tipo de lesión. Aunque las diferencias estadísticas entre los grupos de tipo de lesión siguen la línea de otros estudios (Gil y col., 2015) no sucede así cuando se comparan grupos en función del nivel de los jugadores (Granados y col., 2015). Además, se observa una diferencia entre los valores de la mano dominante y la no dominante, cercanos al $20 \%$. Aunque la explicación pueda parecer obvia debido al uso de la raqueta con la mano dominante, se hace necesario apuntar que los jugadores deben de agarrar y empujar el aro de la silla con ambas manos, y si la fuerza no se realiza de forma simétrica, no se mantendrá una dirección lineal en el desplazamiento de la silla.

La velocidad media del saque fue de $114,90 \mathrm{~km} \cdot \mathrm{h}^{-1}$. Los jugadores con un mayor nivel competitivo realizaron servicios más rápidos $\left(120 \mathrm{~km} \cdot \mathrm{h}^{-1}\right)$ que los de nivel competitivo más bajo $\left(110,3 \mathrm{~km} \cdot \mathrm{h}^{-1}\right)$ aspecto que también sucede en los jugadores de tenis de pie (Reid, Quinn, y Crespo, 2003). No se encontraron diferencias estadísticamente significativas ni entre el nivel competitivo ni por el tipo de lesión, aunque en ambos casos se mostró un tamaño del efecto superior a 1 punto considerado como un tamaño del efecto alto (Rhea, 2004). Las diferencias en el grupo del tipo de lesión siguen la línea del estudio de Cavedon y col. (2014) realizado en jugadores de TSR, donde se muestra que los jugadores con una LM tienen valores más bajos que los jugadores con otro tipo de lesiones como poliomielitis o amputaciones. 
Sánchez-Pay, A.; Sanz-Rivas, D. (2019). Evaluación de la condición física del jugador de tenis en silla de ruedas de alto nivel según nivel competitivo y tipo de lesión. RICYDE. Revista internacional de ciencias del deporte. 57(15), 235-248. https://doi.org/10.5232/ricyde2019.05702

La capacidad que tienen los jugadores de acelerar rápido y de cambiar de dirección parecen ser muy importantes en el TSR (Bullock y Pluim, 2003; Goosey-Tolfrey y Moss, 2005). Los jugadores de TSR de alto nivel mostraron un menor tiempo en recorrer cada tramo (5-10-20 $\mathrm{m}$ ) con y sin raqueta, que los jugadores de nivel más bajo (tabla 3). Además, mostraron diferencias estadísticamente significativas en casi todos los tramos. Los valores para $5 \mathrm{~m}$ fueron ligeramente menores a los encontrados en jugadores de baloncesto en silla de ruedas $(1,57 \pm 0,14$ vs $1,72 \pm 0,08$ s) (Yanci y col., 2015), en cambio, los jugadores de TSR muestran valores más altos en la distancia de $20 \mathrm{~m}(5,62 \pm 0,64$ vs 5,16 $\pm 0,18 \mathrm{~s})$, quizá debido a que los jugadores de TSR no recorren distancias tan largas en una pista de tenis como ocurre en un campo de baloncesto. Comparando los datos en función del tipo de lesión (tabla 4), se observa que el tiempo es mayor en todos los parciales para los jugadores con una $\mathrm{LM}$, con un tamaño del efecto superior a 1 punto excepto en $10 \mathrm{~m}$ sin raqueta $\left(\mathrm{d}_{\mathrm{g}}=0.96\right)$; si bien, sólo se encontraron diferencias estadísticamente significativas en el tramo de $5 \mathrm{~m}$ con raqueta $(\mathrm{p}=.010)$ y $20 \mathrm{~m}(\mathrm{p}=.037)$. Aunque el grupo de $\mathrm{LM}$ es heterogéneo en lo que a nivel de afectación funcional se refiere (afectación a nivel lumbar, a nivel dorsal baja y dorsal alta) es conocido que la estabilidad del tronco tiene una relación directa con la capacidad de acelerar la silla (Goosey-Tolfrey y Moss, 2005). En el ciclo de empuje a los aros de las ruedas de la silla, el jugador debe utilizar el tronco con una flexión del mismo para que los brazos tengan un mayor tiempo para imprimir fuerza al aro (Vanlandewijck, Theisen, y Daly, 2001). Jugadores con una afectación medular más alta tendrán menor capacidad para utilizar los músculos flexores y extensores del tronco que los jugadores con una afectación medular más baja o incompleta (Goosey-Tolfrey y Leicht, 2013; Hoffman, 1986). Este hecho puede comprometer la eficiencia de las acciones sobre la silla y explicar, en cierto modo, la diferencia de valores tanto a nivel funcional como por grupo de nivel.

El jugador de TSR debe realizar aceleraciones y giros tras cada golpe, por lo que evaluar la capacidad de cambiar de dirección del jugador se hace necesaria. El T-test con uso de raqueta mostró mejores valores para los jugadores con mejor ranking que los de peor ranking (tabla $2)$, mostrando diferencias estadísticamente significativas $\left(\mathrm{p}=.034, \mathrm{~d}_{\mathrm{g}}=1,57\right)$ tal y como se ha mostrado con jugadores de baloncesto de diferente nivel (Granados y col., 2015). Este test incluye aceleraciones, frenadas y giros, que son las acciones que debe realizar el jugador de TSR durante el punto (Sanz, 2003). Realizarlo en el menor tiempo posible permitirá al jugador llegar con mejor posición para golpear la pelota y girar rápidamente para volver a estar preparado para la siguiente acción. Estas diferencias en la velocidad de aceleración, frenado y giro pueden tener la explicación en un mayor nivel de experiencia de los jugadores, realizando movimientos más eficientes con mayores fuerzas de propulsión (Gorce y Louis, 2012). El T-Test con raqueta también muestra diferencias estadísticamente significativas ( $\mathrm{p}=$ $.041, \mathrm{~d}_{\mathrm{g}}=1,49$ ) cuando se comparan los grupos del tipo de lesión (tabla 3). Aunque los valores no pueden ser comparados entre diferentes estudios debido a que las distancias de la prueba son diferentes entre ambos, la diferencia entre grupos del tipo de lesión también ha sido observado en jugadores de baloncesto con y sin LM (Gil y col., 2015). Los continuos giros que realizan los jugadores sobre la silla produce modificaciones del centro de masas así como el centro de presiones (Bascou y col., 2012). Es por ello que para mantener la estabilidad del conjunto jugador-silla, los deportistas usan correas y anclajes para ajustar las diferentes partes y extremidades del cuerpo a la silla.

En las pruebas de lanzamiento de balón medicinal se observa que los jugadores con mayor nivel competitivo tienen unos valores superiores de distancia de lanzamiento que los jugadores con menor nivel competitivo (tabla 2). Esto también sucede cuando se compara en función del nivel funcional de los jugadores (tabla 3), mostrando un tamaño del efecto superior a 1 punto en las tres pruebas. El lanzamiento simulando un servicio es 
Sánchez-Pay, A.; Sanz-Rivas, D. (2019). Evaluación de la condición física del jugador de tenis en silla de ruedas de alto nivel según nivel competitivo y tipo de lesión. RICYDE. Revista internacional de ciencias del deporte. 57(15), 235-248. https://doi.org/10.5232/ricyde2019.05702

estadísticamente mayor $\left(\mathrm{p}=.029, \mathrm{~d}_{\mathrm{g}}=1,64\right)$ para los jugadores con mejor ranking que para los peor clasificados. Esto podría explicar las diferencias en la velocidad de servicio entre ambos grupos, pues existe similitud de movimientos entre el lanzamiento del balón y el gesto técnico del saque, habiendo sido utilizado como ejercicio de transferencia al gesto técnico (Roetert, Ellenbecker, y Reid, 2009).

El TSR es un deporte de carácter intermitente, con intervalos de trabajo de 15-20\% sobre el tiempo total de un partido, con una intensidad catalogada como media/alta (Sánchez-Pay y col. 2015). En la bibliografía existen diferentes pruebas para la valoración de la resistencia de un deportista en silla de ruedas (Vanderthommen, Francaux, Colinet y col., 2002; Vanlandewijck, Van De Vliet, Verellen, y Theisen, 2006) y sólo una específica de TSR (De Groot, Valent, Fickert, Pluim, y Houdijk, 2016). Aunque el test desarrollado por De Groot y col. (2016) es de intensidad incremental, éste posee un carácter continuo, con desplazamientos de $20 \mathrm{~m}$, sin necesidad de utilizar la raqueta ni de simular un golpeo, aspectos que muestran la falta de especificidad del test al deporte de TSR. En este estudio, los jugadores de TSR mostraron unos valores promedio ligeramente superior a 15 palieres, valores similares a los encontrados en tenistas adultos de pie (Ferrauti y col., 2011). Los jugadores de alto nivel consiguieron llegar hasta el palier 18, mientras que los de nivel más bajo llegaron al 13, mostrando diferencias estadísticamente significativas $\left(\mathrm{p}=.002, \mathrm{~d}_{\mathrm{g}}=\right.$ $2.94)$, aspecto que también se observa cuando se comparara en función del tipo de lesión ( $\mathrm{p}=$ $\left..034, \mathrm{~d}_{\mathrm{g}}=1,57\right)$.

\section{Conclusiones}

En líneas generales, los resultados de este estudio muestran que los jugadores con un mejor ranking tienen mejor nivel de condición física que los jugadores con un ranking más bajo. Realizan servicios más veloces, aceleran y giran con la silla más rápido, tienen mejores valores en los lanzamientos de balón medicinal, así como una mejor resistencia específica en pista. Que los jugadores posean una LM parece afectar a los valores de los test, aunque no existen tantas diferencias como en la comparación de grupos de nivel. Es necesario destacar, que los datos aquí obtenidos evalúan el jugador-silla en su conjunto. Así, se conoce que la configuración de la silla afecta tanto a la cinemática de la parte superior del cuerpo (Gorce y Louis, 2012), como a la velocidad de movimiento (Kotajarvi, Sabick, An, Shao, Kaufman, y Basford, 2004). Dentro de esta configuración, los jugadores modifican el peso y tamaño de la silla, la altura del asiento y del respaldo, el diámetro de las ruedas y la inclinación de las mismas, el uso de cinchas en función del tipo de lesión y afectación funcional, etc. La configuración optima para cada jugador es personal, y estos aspectos no han sido tenidos en cuenta en este estudio.

Las pruebas utilizadas en este estudio parecen ser aplicables a jugadores de TSR por dos motivos. Primero, los jugadores con mejor ranking obtienen mejores valores en todas las pruebas, obteniendo diferencias estadísticamente significativas en más de la mitad de las mismas, por lo que estos test parecen discriminar entre diferentes grupos de nivel. Segundo, aunque sea obvio que los jugadores con una lesión medular tengan peores valores en todas las pruebas de condición física, sólo se encontraron diferencias estadísticamente significativas en 5 de las 15 pruebas, por lo que podemos decir que estos tests comúnmente utilizados en el tenis convencional no son excluyentes en función del tipo de lesión. 
Sánchez-Pay, A.; Sanz-Rivas, D. (2019). Evaluación de la condición física del jugador de tenis en silla de ruedas de alto nivel según nivel competitivo y tipo de lesión. RICYDE. Revista internacional de ciencias del deporte. 57(15), 235-248. https://doi.org/10.5232/ricyde2019.05702

Aunque el estudio posee algunas limitaciones como el pequeño tamaño de la muestra, así como no haber realizado un mayor número de grupos en función del tipo de lesión o limitación funcional, cuenta con los mejores jugadores nacionales y con una alta clasificación internacional. Los valores mostrados en este estudio pueden ser tomados como referencia por entrenadores y preparadores físicos de jugadores en silla de ruedas en general, y de tenistas en silla de ruedas en particular. Los autores indican la necesidad de realizar este tipo de pruebas en la categoría femenina, así como poder establecer diferencias entre las categorías Open y Quad de tenis en silla de ruedas.

\section{Referencias}

Abel, T.; Platen, P.; Rojas Vega, S.; Schneider, S., y Strüder, H. K. (2008). Energy expenditure in ball games for wheelchair users. Spinal Cord, 46(12), 785-790. https://doi.org/10.1038/sc.2008.54

Almena, A.; Pérez-Tejero, J.; Coterón, J., y Veiga, S. (2015). Análisis de la competición en la prueba de 100 metros estilo libre en nadadores españoles con discapacidad física: influencia de la clasificación funcional. Revista de Entrenamiento Deportivo, 29(2), 1-8.

Bascou, J., Sauret, C., Pillet, H., Bonnefoy, A., Thoreux, P., y Lavaste, F. (2012). Evolutions of the wheelchair user's centre of mass and centre of pressure according to the seat fore-aft position during sprinting: a case study of an elite wheelchair tennis player. Computer Methods in Biomechanics and Biomedical Engineering, 15(sup1), 210-211.

Bernardi, M.; Guerra, E.; Di Giacinto, B.; Di Cesare, A.; Castellano, V., y Bhambhani, Y. (2010). Field evaluation of paralympic athletes in selected sports: Implications for training. Medicine and Science in Sports and Exercise, 42(6), 1200-1208. https://doi.org/10.1249/MSS.0b013e3181c67d82

Bullock, M., y Pluim, B. (2003). Wheelchair tennis and physical conditioning. ITF Wheelchair Tennis Coaches Review, 3(9), 2-10.

Cavedon, V.; Zancanaro, C., y Milanese, C. (2014). Kinematic analysis of the wheelchair tennis serve: Implications for classification. Scandinavian Journal of Medicine and Science in Sports, 24(5), 381-388. https://doi.org/10.1111/sms.12182

Croft, L.; Dybrus, S.; Lenton, J., y Goosey-Tolfrey, V. L. (2010). A comparison of the physiological demands of wheelchair basketball and wheelchair tennis. International Journal of Sports Physiology and Performance, 5(3), 301-15.

https://doi.org/10.1123/ijspp.5.3.301

De Groot, S.; Balvers, I. J. M.; Kouwenhoven, S. M., y Janssen, T. W. J. (2012). Validity and reliability of tests determining performance-related components of wheelchair basketball. Journal of Sports Sciences, 30(9), 879-887.

https://doi.org/10.1080/02640414.2012.675082

De Groot, S.; Valent, L. J.; Fickert, R.; Pluim, B. M., y Houdijk, H. (2016). An incremental shuttle wheel test for wheelchair tennis players. International Journal of Sports Physiology and Performance, 11(8), 1111-1114. https://doi.org/10.1123/ijspp.2015-0598

Diaper, N. J., y Goosey-Tolfrey, V. L. (2009). A physiological case study of a paralympic wheelchair tennis player: Reflective practise. Journal of Sports Science and Medicine, $8(2), 300-307$. 
Sánchez-Pay, A.; Sanz-Rivas, D. (2019). Evaluación de la condición física del jugador de tenis en silla de ruedas de alto nivel según nivel competitivo y tipo de lesión. RICYDE. Revista internacional de ciencias del deporte. 57(15), 235-248. https://doi.org/10.5232/ricyde2019.05702

Ferrauti, A.; Kinner, V., y Fernandez-Fernandez, J. (2011). The hit \& turn tennis test: An acoustically controlled endurance test for tennis players. Journal of Sports Sciences, 29(5), 485-494.

https://doi.org/10.1080/02640414.2010.539247

Filipčič, T., y Filipčič, A. (2009a). Analysis of movement velocity and distance covered in wheelchair tennis. Kinesiologia Slovenica, 32, 25-32.

Filipčič, T., y Filipčič, A. (2009b). Time characteristics in wheelchair tennis played on hard surfaces. Kinesiology, 41(1), 67-75.

Gil, S. M.; Yanci, J.; Otero, M.; Olasagasti, J.; Badiola, A.; Bidaurrazaga-Letona, I. ; ... y Granados, C. (2015). The functional classification and field test performance in wheelchair basketball players. Journal of Human Kinetics, 46(1), 219-230. https://doi.org/10.1515/hukin-2015-0050.

Goosey-Tolfrey, V. L., y Leicht, C. A. (2013). Field-based physiological testing of wheelchair athletes. Sports Medicine, 43(2), 77-91. https://doi.org/10.1007/s40279-012-0009-6

Goosey-Tolfrey, V. L., y Moss, A. D. (2005). Wheelchair velocity of tennis players during propulsion with and without the use of racquets. Adapted Physical Activity Quarterly, 22, 291-301.

Gorce, P., y Louis, N. (2012). Wheelchair propulsion kinematics in beginners and expert users: Influence of wheelchair settings. Clinical Biomechanics, 27(1), 7-15. https://doi.org/10.1016/j.clinbiomech.2011.07.011

Granados, C.; Yanci, J.; Badiola, A.; Iturricastillo, A.; Otero, M.; Olasagasti, J. ; ... Gil, S. M. (2015). Anthropometry and performance in wheelchair basketball. Journal of Strength and Conditioning Research, 29(7), 1812-1820. https://doi.org/10.1519/JSC.0000000000000817

Hedges L. V., y Olkin I. (1985). Statistical methods for meta-analysis. San Diego CA: Academic Press.

Hoffman, M. D. (1986). Cardiorespiratory fitness and training in quadriplegics and paraplegics. Sports Medicine, 3(5), 312-330. https://doi.org/10.2165/00007256-198603050-00002

Kotajarvi, B. R.; Sabick, M. B.; An, K.-N.; Zhao, K. D.; Kaufman, K. R., y Basford, J. R. (2004). The effect of seat position on wheelchair propulsion biomechanics. Journal of Rehabilitation Research and Development, 41(3B), 403-414. https://doi.org/10.1682/JRRD.2003.01.0008

Mason, B. S.; Van Der Woude, L. H. V., y Goosey-Tolfrey, V. L. (2013). The ergonomics of wheelchair configuration for optimal performance in the wheelchair court sports. Sports Medicine, 43, 23-38. https://doi.org/10.1007/s40279-012-0005-x

Ponzano, M., y Gollin, M. (2017). Physical demand of wheelchair tennis match-play on hard courts and clay courts. International Journal of Performance Analysis in Sport, 17(4), 656-665.

Reid, M.; Quinn, A., y Crespo, M. (2003). Strength and conditioning for tennis. London: International Tennis Federation.

Rhea, M. R. (2004). Determining the magnitude of treatment effects in strength training research through the use of the effect size. Journal of strength and conditioning research, 18, 918-920.

Roeter, P., y Ellenbecker, T. (2008). Preparación Física Completa para el Tenis. Madrid: Tutor. 
Sánchez-Pay, A.; Sanz-Rivas, D. (2019). Evaluación de la condición física del jugador de tenis en silla de ruedas de alto nivel según nivel competitivo y tipo de lesión. RICYDE. Revista internacional de ciencias del deporte. 57(15), 235-248. https://doi.org/10.5232/ricyde2019.05702

Roetert, E. P.; Ellenbecker, T. S., y Reid, M. (2009). Biomechanics of the tennis serve: Implications for strength training. Strength and Conditioning Journal, 31(4), 35-40. https://doi.org/10.1519/SSC.0b013e3181af65e1

Roy, J. L. P.; Menear, K. S.; Schmid, M. M. A, Hunter, G. R., y Malone, L. A. (2006). Physiological responses of skilled players during a competitive wheelchair tennis match. Journal of Strength \& Conditioning Research, 20(3), 665-671. https://doi.org/10.1519/R-17845.1

Sanchez-Pay, A.; Torres-Luque, G.; Cabello Manrique, D.; Sanz-Rivas, D., y Palao, J. M. (2015). Match analysis of women's wheelchair tennis matches for the Paralympic Games. International Journal of Performance Analysis in Sport, 15(1), 69-79.

Sánchez-Pay, A.; Torres-Luque, G.; Fernandez-García, Á. I.; Sanz-Rivas, D., y Palao, J. M. (2017). Differences in game statistics between winning and losing for male wheelchair tennis players in Paralympics Games. Motriz: Revista de Educação Física, 23(3), 1-6.

Sánchez-Pay, A.; Torres-Luque, G., y Palao, J. M. (2011). Revisión y análisis de los test físicos empleados en tenis. European Journal of Human Movement, 26, 105-122.

Sánchez-Pay, A.; Torres-Luque, G., y Sanz-Rivas, D. (2015). Match activity and physiological load in wheelchair tennis players: a pilot study. Spinal Cord, 54, 229233. https://doi.org/10.1038/sc.2015.107

Sanz, D. (2003). El tenis en silla de ruedas. Barcelona: Paidotribo.

Schuster, J.; Howells, D.; Robineau, J.; Couderc, A.; Natera, A.; Lumley, N.; ... Winkelman, N. (2018). Physical preparation recommendations for elite rugby sevens performance. International Journal of Sports Physiology and Performance, 13(3), 255-268. https://doi.org/10.1123/ijspp.2016-0728

Vanderthommen, M.; Francaux, M.; Colinet, C.; Lehance, C.; Lhermerout, C.; Crielaard, J.-M., y Theisen, D. (2002). A multistage field test of wheelchair users for evaluation of fitness and prediction of peak oxygen consumption. Journal of Rehabilitation Research and Development, 39(6), 685-692.

Vanlandewijck, Y. C.; Evaggelinou, C.; Daly, D. J.; Verellen, J.; Van Houtte, S.; Aspeslagh, V.; ... Zwakhoven, B. (2004). The relationship between functional potential and field performance in elite female wheelchair basketball players. Journal of Sports Sciences, 22(7), 668-675.

https://doi.org/10.1080/02640410310001655750

Vanlandewijck, Y. C.; Van De Vliet, P.; Verellen, J., y Theisen, D. (2006). Determinants of shuttle run performance in the prediction of peak $\mathrm{VO} 2$ in wheelchair users. Disability and Rehabilitation, 28(20), 1259-1266. https://doi.org/10.1080/09638280600554769

Vanlandewijck, Y.; Theisen, D., y Daly, D. (2001). Wheelchair propulsion biomechanics: implications for wheelchair sports. Sports Medicine, 31(5), 339-367. https://doi.org/10.2165/00007256-200131050-00005

Yanci, J.; Iturricastillo, A.; Lozano, L., y Granados, C. (2015). Análisis de la condición física de jugadores nacionales de baloncesto en silla atendiendo a la clasificación funcional. RICYDE. Revista Internacional de Ciencias del Deporte, 11(40), 173-185. https://dx.doi.org/10.5232/ricyde2015.04006 